\title{
TID and Displacement Damage Effects in Vertical and Lateral Power MOSFETs for Integrated DC-DC Converters
}

\author{
Federico Faccio, B. Allongue, G. Blanchot, C. Fuentes, S. Michelis, S. Orlandi, and R. Sorge
}

\begin{abstract}
TID and displacement damage effects are studied for vertical and lateral power MOSFETs in five different technologies in view of the development of radiation-tolerant fully integrated DC-DC converters. Investigation is pushed to the very high level of radiation expected for an upgrade to the LHC experiments. TID induces threshold voltage shifts and, in n-channel transistors, source-drain leakage currents. Wide variability in the magnitude of these effects is observed. Displacement damage increases the on-resistance of both vertical and lateral high-voltage transistors. In the latter case, degradation at high particle fluence might lead to a distortion of the output characteristics curve. HBD techniques to limit or eliminate the radiation-induced leakage currents are successfully applied to these high-voltage transistors, but have to be used carefully to avoid consequences on the breakdown voltage.
\end{abstract}

Index Terms-DC-DC converter, displacement damage, LDMOS, radiation effects.

\section{INTRODUCTION}

W HILE the Large Hadron Collider (LHC), the largest particle accelerator ever built, is just starting its operation at CERN, the upgrade of the accelerator and the associated Physics Detector systems is already being planned. The upgraded detectors will require an increase in the number of electronic readout channels, demanding more power to be distributed in the detector system. This should be made without the addition of material in the detector volume, because of lack of physical space to run more cables and-more importantly-because material in this space is detrimental to the physics performance of the detector. A possible solution relies on the distribution of power at higher voltage (10-12 V) with local DC-DC conversion on-detector to the $1.2 \mathrm{~V}$ required by the front-end electronics. This implies the availability of DC-DC converters

Manuscript received September 11, 2009; revised November 19, 2009; accepted November 19, 2009. Date of current version August 18, 2010. S. Michelis was supported by a Marie Curie Early Stage Research Training of the European Community's 6th Framework Programme under contract number MEST-CT2005-020216-Elacco. C. Fuentes was supported by MECESUP-Chile under grant FSM0601.

F. Faccio, B. Allongue, G. Blanchot, and S. Orlandi are with CERN, PH Department, 1211 Geneva 23, Switzerland (e-mail: federico.faccio@ cern.ch).

C. Fuentes is with CERN, PH Department, 1211 Geneva 23, Switzerland, and also with UTFSM, Valparaiso, Chile.

S. Michelis is with CERN, PH Department, 1211 Geneva 23, Switzerland, and also with the Department of Electronic Engineering, EPFL, Lausanne, Switzerland.

R. Sorge is with IHP (Innovations for High Performance microelectronics), Im Technologiepark 25, 15236 Frankfurt (Oder), Germany.

Color versions of one or more of the figures in this paper are available online at http://ieeexplore.ieee.org.

Digital Object Identifier 10.1109/TNS.2010.2049584 capable of reliable operation in the radiation and magnetic field environment of the detector.

The radiation tolerance specification for the converter is based on the simulated environment in the central tracker detector over its projected lifetime: up to $250 \mathrm{Mrad}\left(\mathrm{SiO}_{2}\right)$ in Total Ionizing Dose (TID) and $2.5 \cdot 10^{15} \mathrm{n} / \mathrm{cm}^{2}$ (1 MeV neutron equivalent) for displacement damage. The central tracker is the innermost detector, hence the one most exposed to radiation and for which space and material budget requirements are the strictest. Additionally, the converter should be capable of operation in the $4 \mathrm{~T}$ magnetic flux density in the tracker volume. This forbids the use of conventional ferromagnetic cores for the inductors, since they saturate at flux densities below $3 \mathrm{~T}$. Coreless (air-core) inductors have to be used instead, limiting the accessible values of inductance below about 5-700 $\mathrm{nH}$ in order to maintain affordable size and weight [1]. The combined specifications in terms of radiation and magnetic field tolerance call for the development of a custom converter.

A comparative study indicated that the buck converter is one of the most suitable converter topology for the intended application [2]. A simplified view of this converter is shown in Fig. 1. Given the affordable range of the coreless inductor value, switching should occur at high frequency $-1 \mathrm{MHz}$ or more. To efficiently drive the switches at this frequency, and to achieve the highest miniaturization, the full converter should be integrated in a single Application Specific Integrated Circuit (ASIC) with only the inductor and capacitors off-chip. The appropriate semiconductor technology for this ASIC should therefore offer both "core" low-voltage transistors (for the control circuitry) and high-voltage transistors (to be used as power switches and wherever direct connection to the input voltage is needed, such as for the internal regulators).

For a converter with an input voltage of 10-12 V, the high voltage transistors shall be rated to withstand a drain-source voltage $\left(\mathrm{V}_{\mathrm{ds}}\right)$ of $16-20 \mathrm{~V}$ at least. Several technologies offering this combination of core and high voltage devices exist in the marketplace, based on standard mixed-signal CMOS technologies $(0.35-0.13 \mu \mathrm{m}$ node) to which a high-voltage module extension has been added. The radiation effects affecting the low-voltage devices in these CMOS nodes are well known [3]. In particular, these circuits are not sensitive to displacement damage up to the fluence levels of interest for this work. Hardness-By-Design (HBD) techniques allowing to eliminating TID-induced leakage currents are also well known and have been extensively used in the past [4], [5]. For this reason, this work focuses on the high-voltage transistors whose radiation performance is considerably less known. 


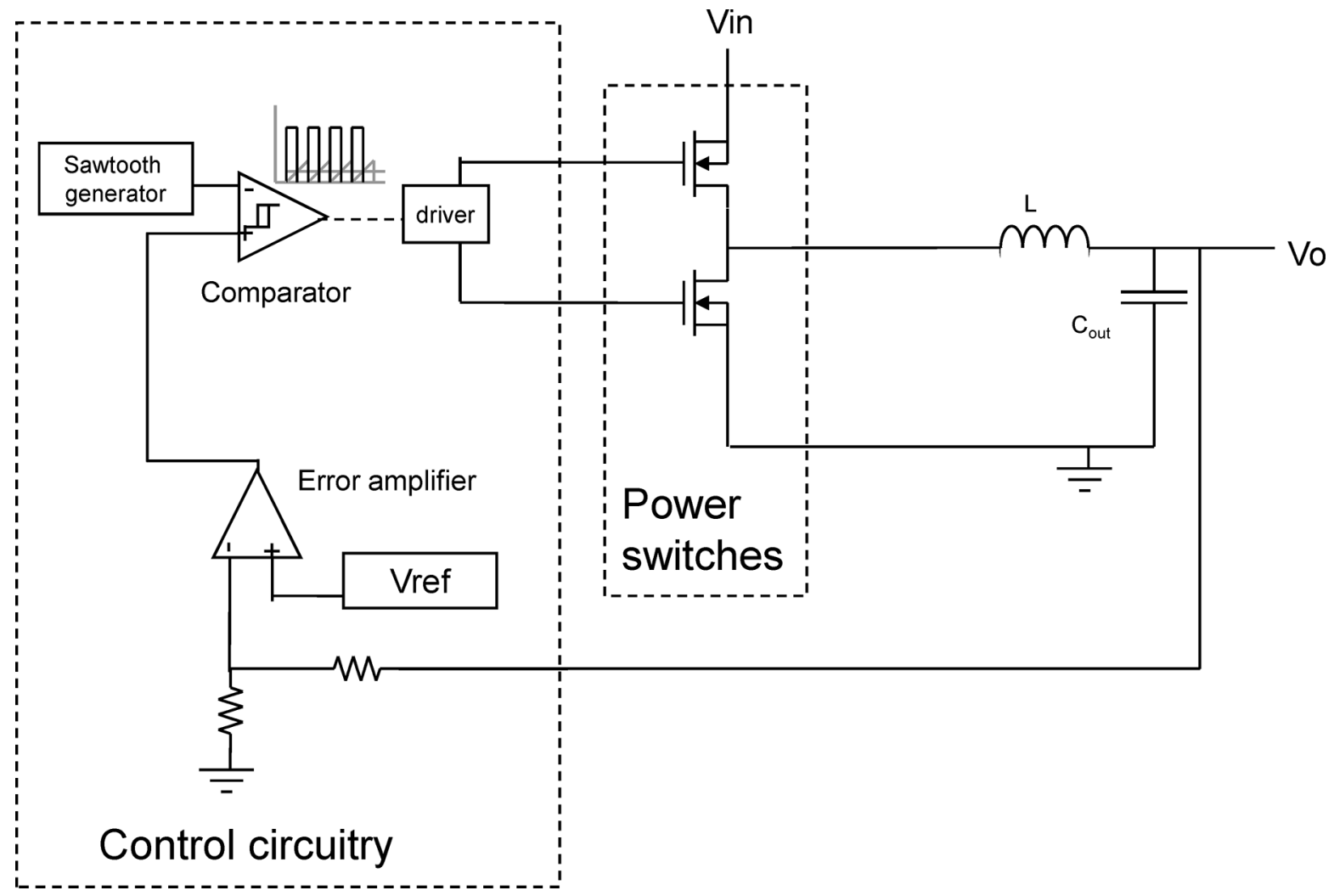

Fig. 1. Simplified architecture of a buck DC-DC converter.

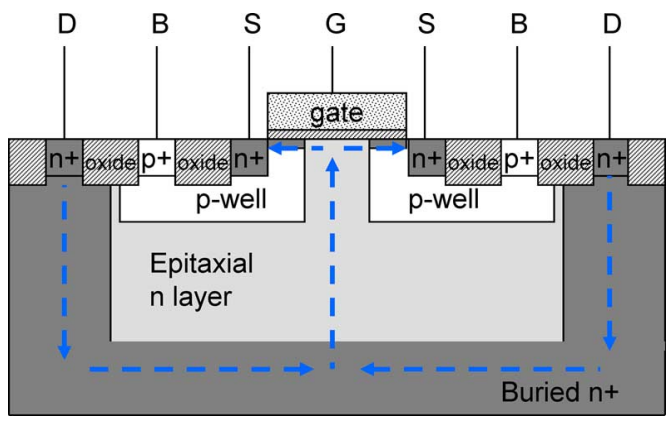

Fig. 2. Schematic view of a vertical NMOS transistor. The current path in the presence of the inversion channel under the gate is indicated by the arrows.

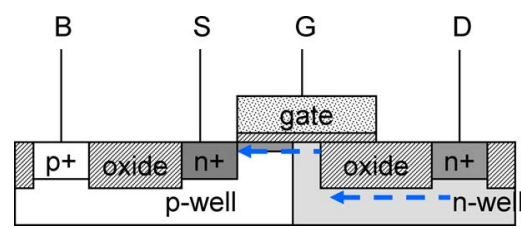

Fig. 3. Schematic view of a LDMOS N-channel transistor. The current path in the presence of the inversion channel under the gate is indicated by the arrows.

With the aim of isolating possible common trends in radiation response, we study TID and displacement damage effects in high-voltage transistors in five technologies available for ASIC design. We also explore for the first time the possibility to increase the radiation tolerance of these high-voltage devices with HBD techniques derived from those commonly used in lowvoltage designs in CMOS. The work on the $0.25 \mu \mathrm{m}$ technology (identified as "B" in this paper) was done in close collaboration with the manufacturer, IHP, who made available samples of high voltage transistors from different development stages in their SGB25VGOD technology. IHP also custom-modified these transistors with HBD techniques and made them available for testing.

\section{EXPERIMENTAL DETAILS}

\section{A. High-Voltage Transistors}

The lithography nodes of the core CMOS in the five technologies studied varied from 0.35 down to $0.13 \mu \mathrm{m}$. The characteristics of the high-voltage transistors in each of them are very variable, since all manufacturers made specific choices leading to different $\mathrm{V}_{\mathrm{gs}}$ or $\mathrm{V}_{\mathrm{ds}}$ ratings, and electrical performance. Large drain-source breakdown voltages are achieved by the use of two different designs [6]: a vertical—used only in one of the explored technologies-and a lateral design. This latter, called LDMOS (Laterally Diffused MOS) is by far the most common and is used in power and RF applications. In a vertical design, schematically shown in Fig. 2, current flows vertically from the heavily doped buried drain to the surface inversion layer under the gate. Large breakdown voltage is achieved via the drift region of the lowly doped epitaxial layer. In the LDMOS, instead, current flows horizontally in a lowly doped drift region as shown schematically in Fig. 3. This design typically achieves smaller on-resistance and capacitance, hence making it more suitable for high-frequency operation. Design choices such as gate overlap over the $\mathrm{n}$ drift region or the presence of the STI oxide in the same area might vary with the technology.

A summary of the main characteristics (type and voltage ratings) of the transistors in the different technologies is presented in Table I. The variability in the LDMOS design (doping levels, 
TABLE I

Properties OF High-VOLTAGE TRANSISTORS IN THE STUdiED TECHNOLOGIES

\begin{tabular}{|c|c|l|c|c|}
\hline Tech. & Tech. node & Transistor type & Max Vds [V] & Max Vgs [V] \\
\hline \multirow{3}{*}{$\mathrm{A}$} & \multirow{3}{*}{$0.35 \mu \mathrm{m}$} & Vertical N & 80 & 3.3 \\
\cline { 3 - 5 } & & LDMOS N & 14 & 3.3 \\
\cline { 3 - 5 } & LDMOS P & 80 & 3.3 \\
\hline \multirow{2}{*}{$\mathrm{B}$} & \multirow{2}{*}{$0.25 \mu \mathrm{m}$} & LDMOS N & 22 & 2.5 \\
\cline { 3 - 5 } & & LDMOS P & 16 & 2.5 \\
\hline $\mathrm{C}$ & $0.18 \mu \mathrm{m}$ & LDMOS N \& P & 20 & 5.5 \\
\hline \multirow{3}{*}{$\mathrm{D}$} & \multirow{3}{*}{$0.18 \mu \mathrm{m}$} & LDMOS N \& P & 20 & 1.8 \\
\cline { 3 - 5 } & & LDMOS N \& P & 50 & 1.8 \\
\cline { 3 - 5 } & & LDMOS N \& P & 25 & 5 \\
\hline $\mathrm{E}$ & $0.13 \mu \mathrm{m}$ & LDMOS N \& P & 20 & 4.5 \\
\hline
\end{tabular}

gate extension, gate oxide thickness, presence of STI oxide over drift region) makes it very difficult to predict the radiation tolerance of the devices a priori.

\section{B. Test Structures and Measurement Procedure}

The test structures were most often made available from the manufacturers as part of their drop-in process monitors in the form of arrays of individual transistors with different dimensions. In most technologies, the LDMOS typically come as predefined cells that can only be stretched in one direction to increase the channel width, the length being fixed to the minimum achievable to have the best performance (typically below $1 \mu \mathrm{m}$ ). Therefore, all samples available had fixed gate length, and gate width ranging from 40 to $5 \mu \mathrm{m}$. Exceptions concerned technologies $\mathrm{D}$ and $\mathrm{E}$, for which the test structures made available by the manufacturer included transistors with much larger gate length (10 and $5 \mu \mathrm{m}$ respectively).

In the 0.35 and $0.25 \mu \mathrm{m}$ technologies, custom-designed test structures were also developed and tested, including both standard and modified vertical and LDMOS devices. The introduced modifications, at the level of the transistor's layout, were inspired by the Enclosed Layout Transistors (ELT) [7] successfully used in low-voltage CMOS. Their aim was the elimination of the source-drain leakage current induced by TID in N-channel transistors. Successful use of these techniques in high-voltage transistors, whose layout critically determines voltage breakdown, has not been demonstrated before.

Measurements of all transistors were performed either under needles at an 8' probe station (Suss Microtech PA200) or on chips mounted on ceramic packages. The terminals of the transistors on-chip were connected to the 6 channels of a semiconductor parameter analyzer HP4145B via a switching matrix Keithley 707. During measurements of each transistor on chip, current was measured as a function of the drain and gate voltage $\left(\mathrm{I}_{\mathrm{ds}}=\mathrm{f}\left(\mathrm{V}_{\mathrm{gs}}, \mathrm{V}_{\mathrm{ds}}\right)\right)$. All measurements, with the exception of those during low-temperature irradiation tests $\left(-30^{\circ} \mathrm{C}\right)$, were performed at room temperature. At least 2 samples of transistors identical or very similar, sometimes rotated by $90^{\circ}$, were always measured on each chip. Results on these samples have always been homogeneous.

\section{Irradiation Procedure}

1) TID: TID measurements took place at the CERN's SEIFERT RP149 X-ray irradiation system. This system produces X-rays peaking at an energy of 10-20 keV by accelerating electrons on a $\mathrm{W}$ target as commonly done for radiation effects studies on semiconductors [8]. Test transistors, either con- nected via the needles of a probe card or mounted in delidded DIL ceramic packages, were always exposed, measured and subsequently annealed at $100{ }^{\circ} \mathrm{C}$ under bias. The most typical bias condition was "Worst Case" (WC), where all terminals of both $\mathrm{N}$ and PMOS transistors were grounded while the gate of the NMOS was kept at the maximum allowed Vdd for the specific gate oxide (highest $\mathrm{E}$ field). In some cases, switched bias conditions replicating at lower frequency the real electrical environment in a DC-DC converter were used: the transistors were turned on-off at $10 \mathrm{kHz}$ while keeping the $\mathrm{V}_{\mathrm{ds}}$ at $12 \mathrm{~V}(-12 \mathrm{~V}$ for the PMOS). Dose rates varied between 20 and $66 \mathrm{krd}\left(\mathrm{SiO}_{2}\right) / \mathrm{min}$, and all measurements were made immediately at the end of each irradiation step.

Temperature during irradiation was typically kept at $27^{\circ} \mathrm{C}$, but measurements in the $0.25 \mu \mathrm{m}$ technology were also made at $-30{ }^{\circ} \mathrm{C}$. This was possible because a thermal chuck, whose temperature is controlled by a custom equipment from DigitConcept (France), is installed at the probe station inside the $\mathrm{X}$-ray irradiation cabinet. Measurements at $-30{ }^{\circ} \mathrm{C}$ are particularly relevant in view of qualifying a technology for our application: the DC-DC converter will be installed in close proximity of the silicon detectors in the SLHC tracker system, which will be kept well below freezing temperature during operation to avoid excessive damage induced by displacement effects in the silicon sensors [9]. Thermal simulations of the final system project operating temperatures of about $-30^{\circ} \mathrm{C}$ for the electronics in this area.

2) Displacement Damage: Proton irradiation took place at the CERN "irrad1" facility, where a $24 \mathrm{GeV} / \mathrm{c}$ proton beam from the PS accelerator is provided for experimental purposes. In this case, samples were exposed naked and without any bias, at room temperature. Exposing naked chips ensures minimal activation of irradiated material, hence producing less radioactive waste and reducing the risk of long-term activation of heavy elements typically used in packages. The lack of bias is not a severe drawback for displacement damage, since the sensitivity of devices to this radiation effect is known not to be dependent on bias (with the exception of photonic devices such as LEDs, lasers and solar cells) [10]. Several samples were exposed, typically in a time span of a few days to a couple of weeks, to reach different proton fluences up to $1 \cdot 10^{16} \mathrm{p} / \mathrm{cm}^{2}$. Given the energy of the proton beam, the 1-MeV neutron equivalent fluence is obtained, with good approximation, by dividing the proton fluence by 2 . It has to be considered that protons also deposit ionizing radiation (TID): a fluence of $1 \cdot 10^{15} \mathrm{p} / \mathrm{cm}^{2}$ corresponds to about $32 \mathrm{Mrad}$ in TID. After irradiation, samples were positioned in a freezer (at about $-27^{\circ} \mathrm{C}$ ) for a period varying between a few days and 2 months. The length of this period was determined by either the need to wait for radioprotection agreement before mounting the samples in DIL packages or the unavailability of the measuring equipment. This waiting time is expected not to affect the results, since defect centers introduced by displacement do not anneal when the samples are cooled to about $-30{ }^{\circ} \mathrm{C}$ [11].

In order to clearly distinguish TID and displacement damage effects, an additional experiment took place at a source of protons of different energy. Some $0.35 \mu \mathrm{m}$ samples were exposed unbiased to the $5 \mathrm{MeV}$ proton beam of Legnaro National Laboratories (LNL), Italy, up to a fluence of $1.3 \cdot 10^{14} \mathrm{p} / \mathrm{cm}^{2}$ (roughly 


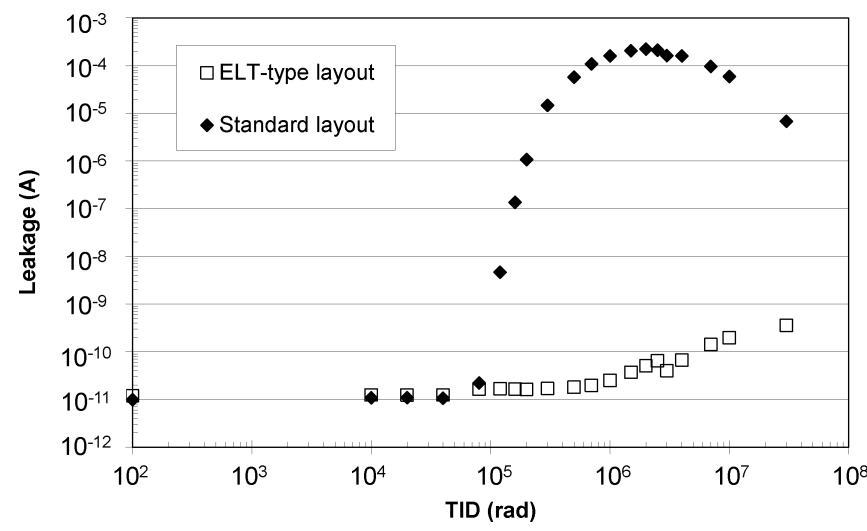

Fig. 4. Drain-source leakage current at $\mathrm{V}_{\mathrm{gs}}=0 \mathrm{~V}$ for vertical NMOS transistors of standard and ELT-type layout as a function of TID up to $80 \mathrm{Mrad}\left(\mathrm{SiO}_{2}\right)$.

corresponding to $5.8 \cdot 10^{14} 1-\mathrm{MeV}$ equivalent neutrons, and a TID of $166 \mathrm{Mrad}$ ) over a period of less than 4 hours. Chips were stored unbiased at room temperature for 6 months before measurement could take place.

\section{RESULTS AND DISCUSSION}

\section{A. Vertical Transistors}

This type of design is only available as n-channel transistors in the $0.35 \mu \mathrm{m}$ technology. Results will hence refer to this device only, which is rated to $3.3 \mathrm{~V}$ maximum $\mathrm{V}_{\mathrm{gs}}$ and $80 \mathrm{~V} \mathrm{~V}$ ds. The gate oxide thickness is the same as for the core CMOS transistors in the technology: about $7 \mathrm{~nm}$.

When exposed to TID, the most notable effect in standard transistors is an increase of the drain-source leakage current at $\mathrm{V}_{\mathrm{gs}}=0 \mathrm{~V}$ occurring already at about $100 \mathrm{krd}\left(\mathrm{SiO}_{2}\right)$ (Fig. 4). The leakage increases sharply with TID reaching a peak around $3 \mathrm{Mrad}(\mathrm{SiO} 2)$, then it decreases with further exposure. Similar behavior has been observed already on low-voltage CMOS transistors, where it was explained with the antagonist contribution of oxide trapped charge and interface states to the threshold voltage shift of the lateral parasitic transistor (at the lateral STI oxide) [12], [13].

The use of an ELT-type layout eliminates this problem efficiently, as shown in Fig. 4. The residual observed increase in the leakage after a TID of $30 \mathrm{Mrad}(\mathrm{SiO} 2)$ is due to the $\mathrm{V}_{\text {th }}$ shift $(-50 \mathrm{mV})$ and the subthreshold swing increase ( 86 to $92 \mathrm{mV} / \mathrm{dec}$ ) of the main transistor. Additional performance degradations are limited to $-20 \%$ in mobility and $+10 \%$ in on-resistance during both irradiation up to $30 \mathrm{Mrad}(\mathrm{SiO} 2)$ and annealing. This demonstrates for the first time that HBD layout techniques can successfully be used to improve the TID tolerance of high-voltage vertical transistors.

Displacement damage affects the transistors much more severely, as shown in Fig. 5 for the output characteristic of samples irradiated with both the PS and LNL proton beams.

The comparable damage for the LNL irradiation and the PS irradiation at $9 \cdot 10^{14} \mathrm{p} / \mathrm{cm}^{2}$ indicates displacement damage, since the NIEL of the two exposures is very comparable $\left(4.5-5.8 \mathrm{n} / \mathrm{cm}^{2} 1 \mathrm{MeV}\right.$ equivalent), while the TID is very different (166 and 29 Mrad respectively). In Fig. 5, the drain

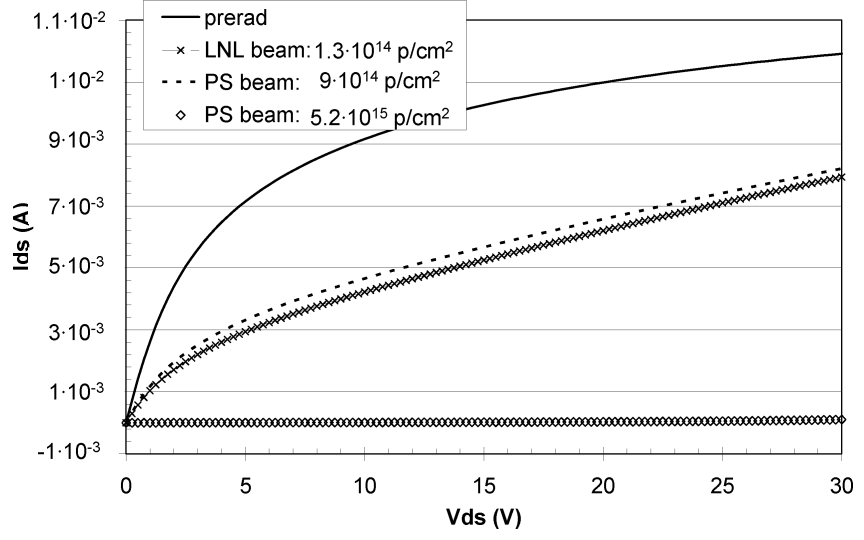

Fig. 5. Displacement damage effect on the output conductance $(\mathrm{Ids}=\mathrm{f}(\mathrm{Vds}))$ of vertical NMOS transistors. $\mathrm{V}_{\mathrm{gs}}=3.3 \mathrm{~V}$.

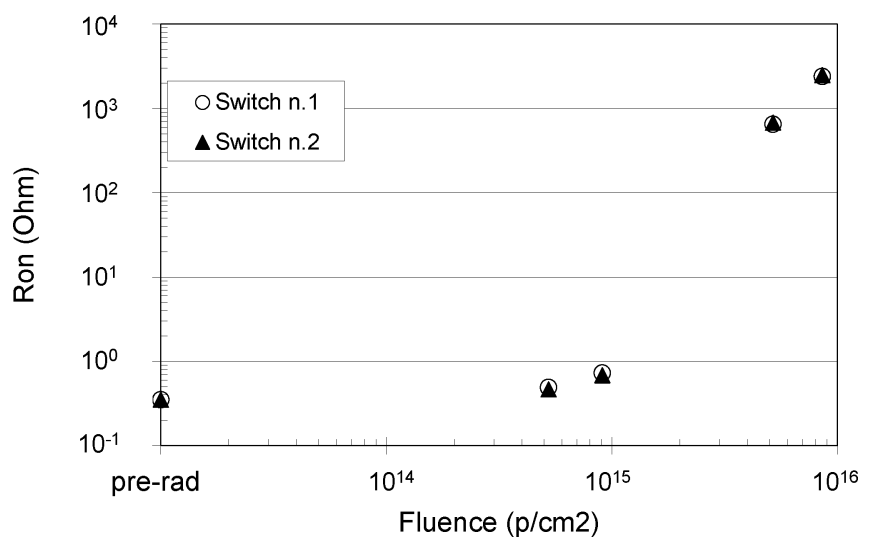

Fig. 6. Increase in the on-resistance of large vertical ELT NMOS transistors with proton fluence (PS beam) for 2 identical samples.

to source current reduces significantly with increasing fluence from $9 \cdot 10^{14} \mathrm{p} / \mathrm{cm}^{2}$ to $5.2 \cdot 10^{15} \mathrm{p} / \mathrm{cm}^{2}$, indicating a sharp increase in the output resistance. This increase is better observable on the on-resistance shown in Fig. 6 for custom-designed ELT transistors with size comparable to the one used for the main switches of a DC-DC converter $(\mathrm{W}=10 \mathrm{~cm})$.

From an initial value of $330 \mathrm{~m} \Omega$, the on-resistance increases already by $30-40 \%$ after the first irradiation step $\left(5 \cdot 10^{14} \mathrm{p} / \mathrm{cm}^{2}\right)$ to eventually reach the $\mathrm{k} \Omega$ level. A similar effect was reported in the past for VDMOS power transistors [14], but not yet for modern high-voltage transistors. It can be explained by the increased resistivity of the lowly doped epitaxial drift layer, where defects introduced by displacement damage can significantly affect the carrier mobility. As such, no layout modification can help mitigating the effect, which in fact has been identically observed in standard and HBD devices. Therefore, these transistors are not suitable for our application where displacement damage tolerance to high levels (above $10^{15} \mathrm{n} / \mathrm{cm}^{2}$ ) is required. They are nonetheless well suitable for applications in Space, where ELT-type layout can safely extend the natural TID tolerance to the multi-Mrad level. It should be pointed out that, for these vertical transistors, this layout modification (horizontal) does not interfere with the high voltage structure (vertical) of the device. It will be shown later that this is not necessarily true for the LDMOS design. 

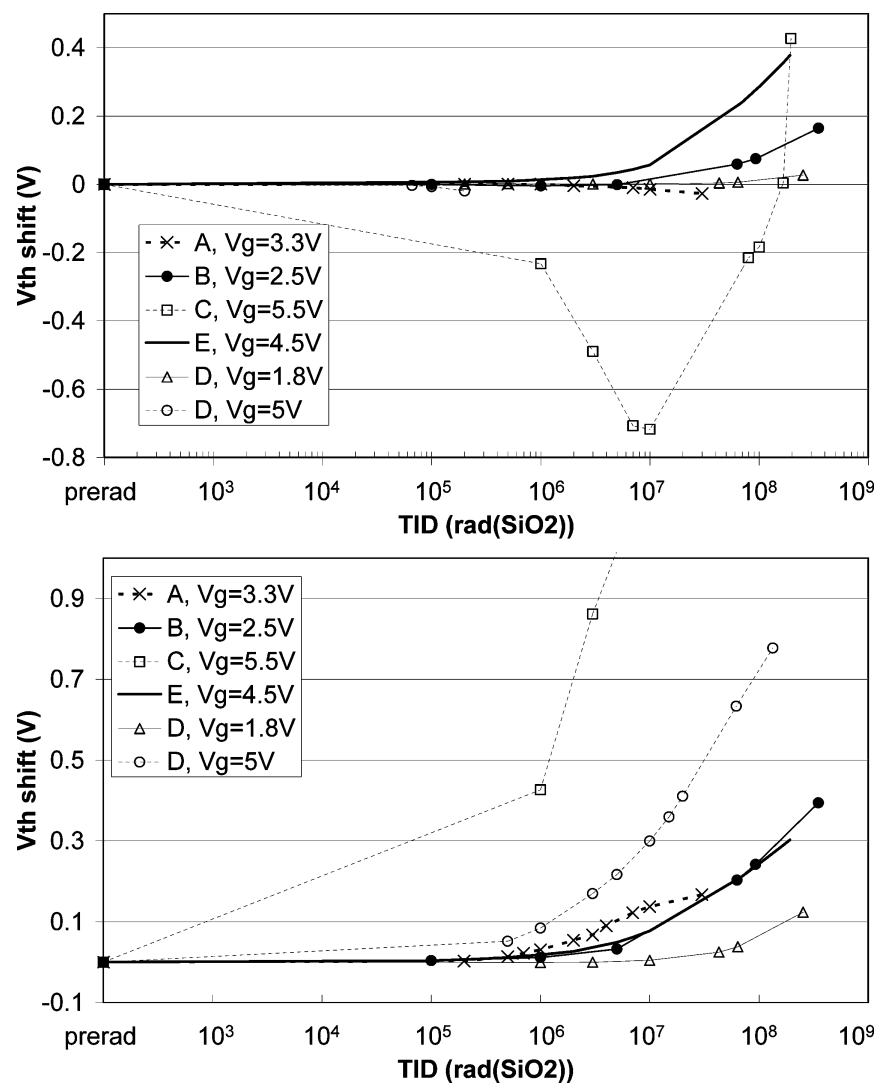

Fig. 7. Threshold voltage shift induced by TID on n-channel (top) and p-channel (bottom) LDMOS transistors biased in the WC condition at room temperature. For the n-channel, technology $\mathrm{D}, \mathrm{Vg}=5 \mathrm{~V}$, the leakage current was too large after $200 \mathrm{krd}\left(\mathrm{SiO}_{2}\right)$ to enable extraction of $\mathrm{V}_{\text {th }}$ in the linear regime.

\section{B. LDMOS Transistors}

1) TID Effects: As already evidenced in the 80s, the TID response of MOS transistors depends strongly on the thickness $t_{o x}$ of the gate oxide [15], [16]: the thinner the oxide, the milder the induced $V_{\text {th }}$ shift. While $t_{\mathrm{ox}}$ decreases with each CMOS generation in the low-voltage world, the same is not true for the LDMOS transistors available in all studied technologies. Depending on the targeted main application for the technology, some manufacturers prefer keeping a thicker oxide to enable larger gate voltage ratings (as in technologies $\mathrm{C}$ and $\mathrm{E}$ ). This might be useful for applications in power management, simplifying the design of products specified for high input voltage. Some manufacturers propose similar devices in addition to their thin oxide offering, providing a large palette of devices to circuit designers (technology $\mathrm{D}$ is an example).

For applications in a radiation environment where TID can exceed $100 \mathrm{Mrad}$, these thick oxide transistors can be penalized by an excessive $V_{\text {th }}$ shift, as indicated by our measurements in Fig. 7 for devices biased in the worst case condition. The increase in $V_{\text {th }}$ affects the on-resistance of the transistor, hence the efficiency of a converter where the transistor is used as power switch. For this reason, it is preferable to use transistors rated to $3.3 \mathrm{~V}$ or less, for which the on-resistance increase with TID up to $250 \mathrm{Mrad}\left(\mathrm{SiO}_{2}\right)$ is typically limited below $50 \%$ for both $\mathrm{n}$ and $\mathrm{p}$-channel transistors in $\mathrm{WC}$ condition.

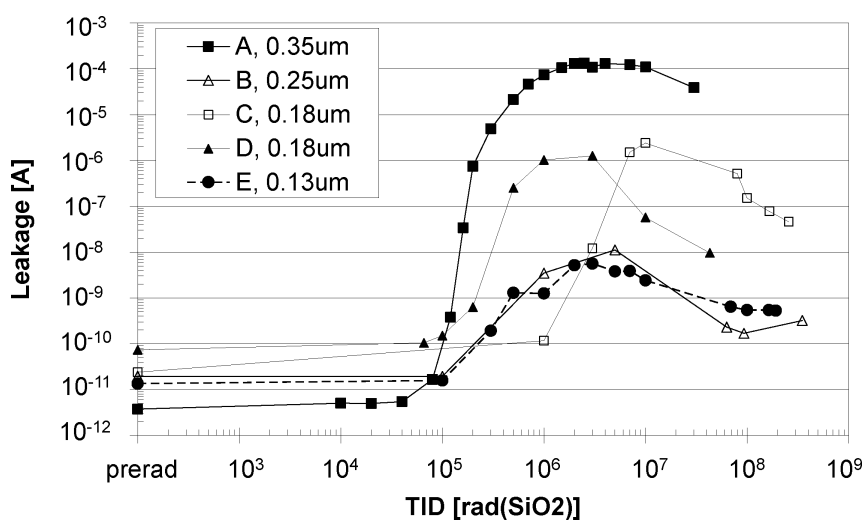

Fig. 8. Evolution of the leakage current with TID for standard (not HBD) $\mathrm{N}$-channel LDMOS in all technologies. Irradiation was performed at room $\mathrm{T}$ under worst case bias. For technology D, data refers to the device rated at $1.8 \mathrm{~V}$.

In analogy to the vertical devices above and to the low-voltage CMOS transistors, the source-drain leakage current of n-channel LDMOS transistors is increased by TID. This effect is determined by charge trapping in the lateral isolation field oxide, typically still used in the construction of LDMOS transistors. Quite interestingly, our measurements in Fig. 8 do not show any systematic correlation between either CMOS technology node, applied gate voltage or $t_{\text {ox }}$ and the leakage current increase. It appears that resilience to TID is rather determined by specific construction choices of every manufacturer (transistor design, channel and substrate doping, oxide growth and deposition conditions), and can not therefore be deduced a priori from the voltage ratings or technology node.

Again, our data show a peak leakage at a TID between $1-10 \mathrm{Mrad}\left(\mathrm{SiO}_{2}\right)$ for all samples. Peak leakage for the IHP quarter micron (B) and for the $0.13 \mu \mathrm{m}$ technologies (E) is surprisingly low when compared to results typically found in literature for low-voltage CMOS transistors [17].

Since the peak leakage is determined by the antagonist contributions of charge trapped in the oxide or in interface states, whose dynamics depend on temperature and applied bias, the leakage evolution also depends on the operating conditions of the transistor. Fig. 9 illustrates how the application of switched bias (on-off switching at $10 \mathrm{kHz}$ ) considerably decreases the peak leakage. Lower temperature, instead, increases it. These data refer to identical transistors from the same lot in the IHP quarter micron technology (B), while the curve in Fig. 8 reports data for a different lot. Some processing parameters were in fact changed between the two lots (the devices were not qualified yet at the time of these measurements).

Due to the annealing of charge trapped in the oxide, the leakage current increase in a real application depends on the dose rate. Our measurements after 1 week annealing at $100{ }^{\circ} \mathrm{C}$ evidence a significant variability in the rate of recovery, as expected from devices with widely different origin. In the impossibility of drawing general conclusions, the real response at different dose rates should be estimated specifically for each technology.

In order to mitigate or completely eliminate the TID- induced leakage in n-channel LDMOS, we have applied HBD layout 


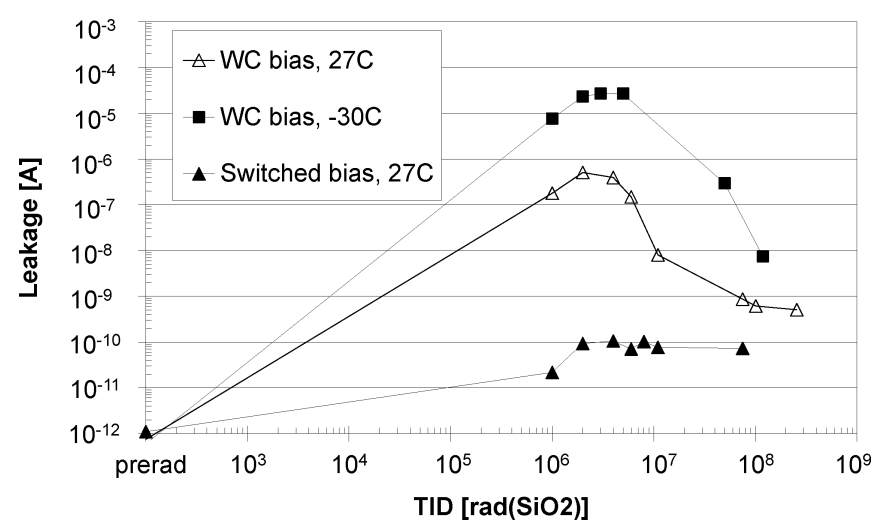

Fig. 9. Influence of the applied bias and temperature during X-ray irradiation for identical transistors in technology B (IHP $0.25 \mu \mathrm{m}$ ).

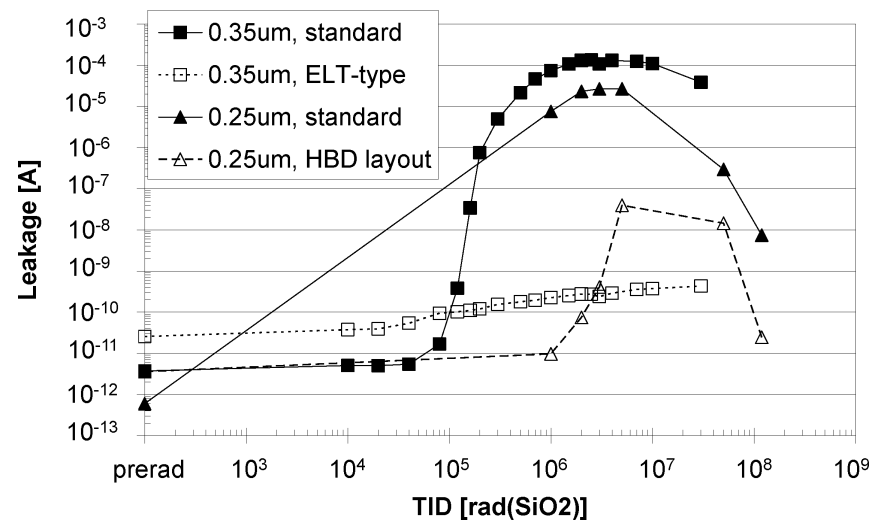

Fig. 10. HBD techniques successfully limit the TID-induced leakage current increase in n-channel LDMOS transistors in two technologies. Measurements on the IHP $0.25 \mu \mathrm{m}$ samples have been taken at the worst case temperature of $-30{ }^{\circ} \mathrm{C}$. Bias was in all cases WC.

techniques and modified in 2 different ways the standard transistors of two of the tested technologies. In the $0.35 \mu \mathrm{m}$ technology (A), the modified design used a technique very similar to the conventional ELT (annular) transistors: a central electrode is surrounded by the gate on all sides. This modification was done by the CERN authors as users of the technology, without consulting with processing engineers from the manufacturer. On the contrary, the modified design in the $0.25 \mu \mathrm{m}$ from IHP adds some distance between the transistor's edges and the STI oxide, this region being covered by thin oxide (and pre-metal dielectric). This modification was directly implemented by process engineers in IHP.

Both modified layouts gave satisfactory results during TID irradiation tests (Fig. 10). The conventional ELT removed the leakage completely, the observed residual increase being attributable to $\mathrm{V}_{\text {th }}$ shift. The alternative design in $0.25 \mu \mathrm{m}$ decreased it by almost 3 orders of magnitude during worst case bias irradiation at $-30{ }^{\circ} \mathrm{C}$ (absolute worst conditions for the leakage current).

2) Displacement Damage Effects: In analyzing the proton irradiation data, it is important to consider that a significant amount of TID accumulates during these tests. Therefore, leakage currents (in $\mathrm{n}$-channel transistors) and $\mathrm{V}_{\text {th }}$ shifts can be observed regularly. The net displacement damage effects
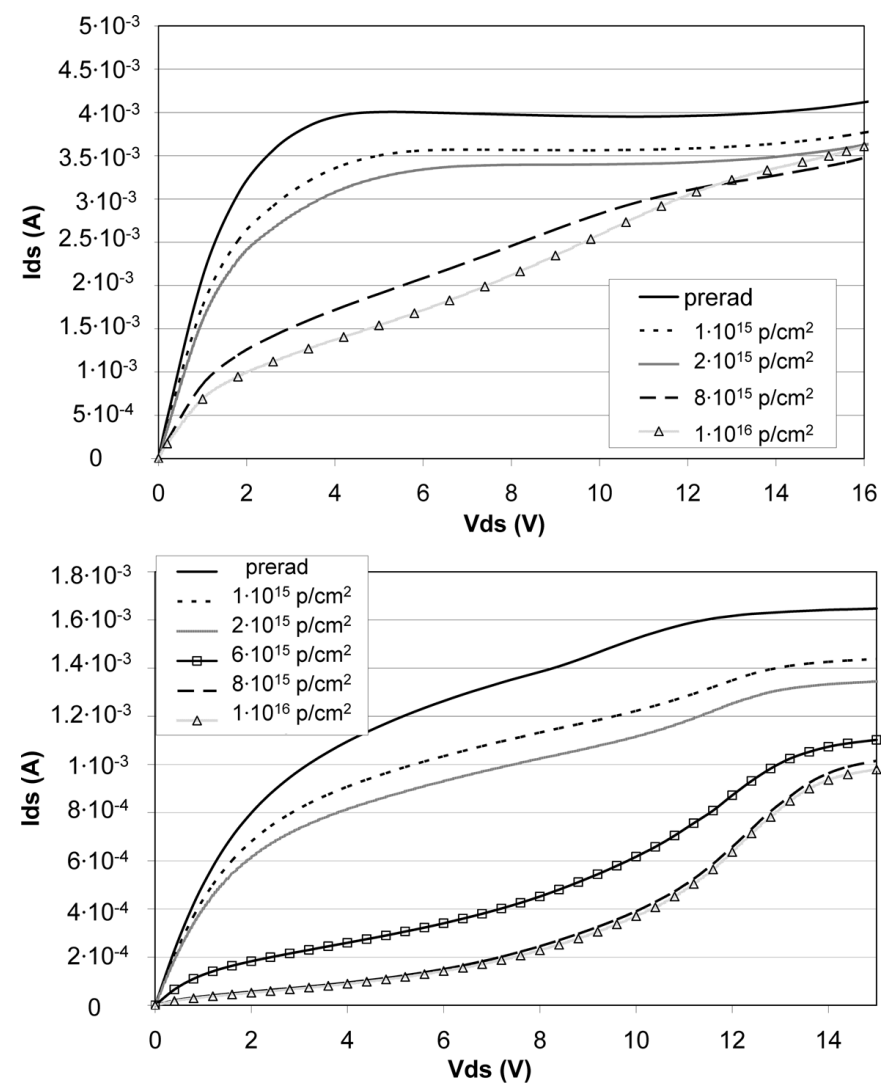

Fig. 11. Evolution of the output characteristics at $\mathrm{Vgs}=2.5 \mathrm{~V}$ with proton irradiation of n-channel (top) and p-channel (bottom) LDMOS transistors in technology B (IHP $0.25 \mu \mathrm{m})$.

described in this section can nonetheless be deduced by the comparison with X-rays irradiation data, where net TID effects can be observed.

LDMOS transistors of both polarities are severely affected by displacement damage, although the onset cumulative fluence for significant degradation widely differs for the technologies under test. The degradation is clearly visible in the output characteristics of the transistors, of which examples are given in Fig. 11 for $\mathrm{n}$ - and p-channel devices in the IHP $0.25 \mu \mathrm{m}$ technology (B).

Measurements after the first irradiation steps show a decrease in the drive current that can be attributed in large part to a TIDinduced increase of the $V_{t h}$. With accumulation of displacement damage, a complete distortion of the output characteristics appears, most noticeably for n-channel LDMOS transistors in all technologies. In some of the studied technologies, the onset of such observable distortion can be as low as $1-2 \cdot 10^{15} \mathrm{p} / \mathrm{cm}^{2}$, as shown in Fig. 12 for the $n$-channel transistor rated at $\mathrm{V}_{\mathrm{gs}}=$ $1.8 \mathrm{~V}$ in one of the $0.18 \mu \mathrm{m}$ processes (D). This relevant modification of the output characteristics indicates that the large number of defects introduced by irradiation in the lowly doped drift region alters the electric field lines in the device. It also determines a very significant increase of the on-resistance, as reported in Fig. 13 for $\mathrm{n}$ - and p-channel transistors in the different technologies studied. In these figures, the large dispersion in the onset fluence for severe performance degradation amongst the different technologies is well apparent. As for the TID effects, also in this case there is no observable correlation between technology node or voltage rating and radiation tolerance. On the 


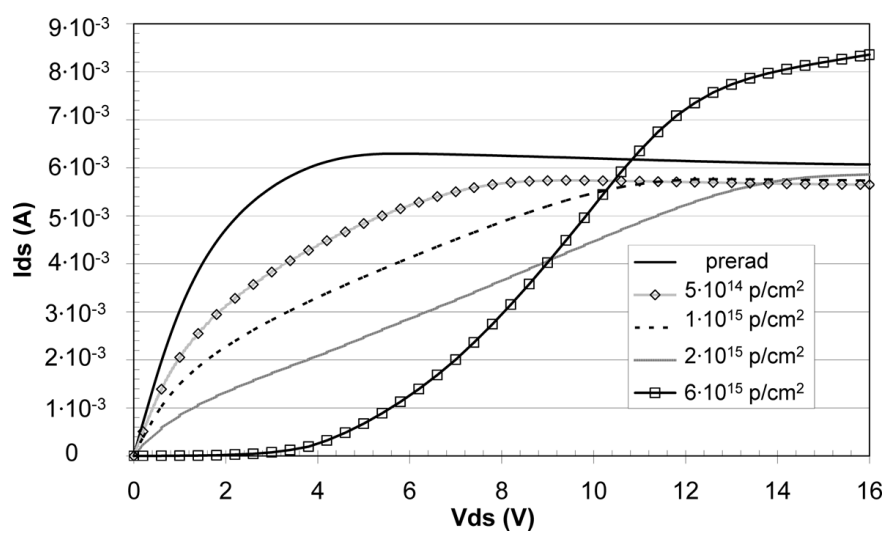

Fig. 12. Evolution of the output characteristics at $\mathrm{Vgs}=1.8 \mathrm{~V}$ with proton irradiation of n-channel LDMOS transistors in technology $\mathrm{D}(0.18 \mu \mathrm{m})$.
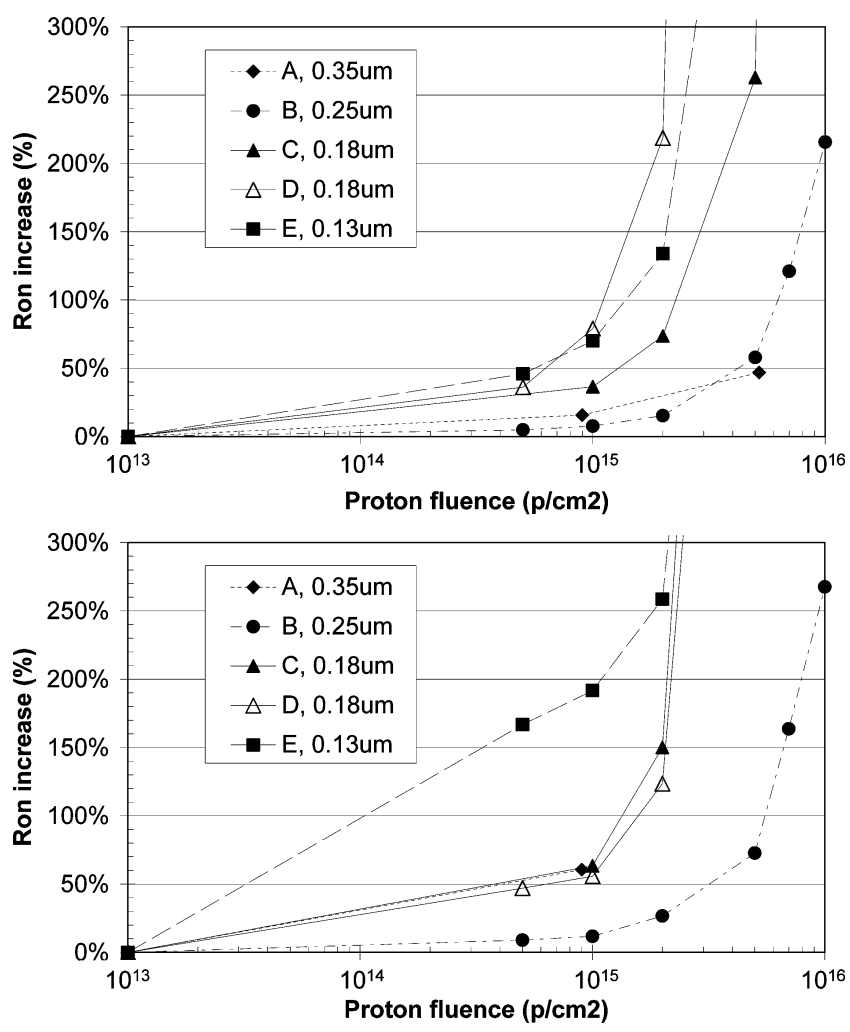

Fig. 13. Increase of the on-resistance of n-channel (top) and p-channel (bottom) LDMOS transistors with proton fluence in the five technologies studied. For technology D, data refers to the device rated at $1.8 \mathrm{~V} \mathrm{Vgs}$ and $\mathrm{V}_{\mathrm{ds}} 20 \mathrm{~V}$ but was comparable for the other available devices. In all cases the maximum rated $\mathrm{Vgs}$ was used in the measurement.

other hand, no annealing of the damage introduced by displacement effects has been observed after annealing at room temperature for long periods (more than 3 months).

Our measurements on transistors with gate length much longer than minimum in technologies $\mathrm{D}(0.18 \mu \mathrm{m})$ and $\mathrm{E}$ $(0.13 \mu \mathrm{m})$ show instead a correlation between gate length and displacement damage. Long-channel transistors degraded considerably less when irradiated with protons, as evidenced in the output characteristics shown in Fig. 14 for technology $\mathrm{D}$, but the n-channel still shows a completely deformed output characteristic curve at the fluence of $6 \cdot 10^{15} \mathrm{p} / \mathrm{cm}^{2}$, being in

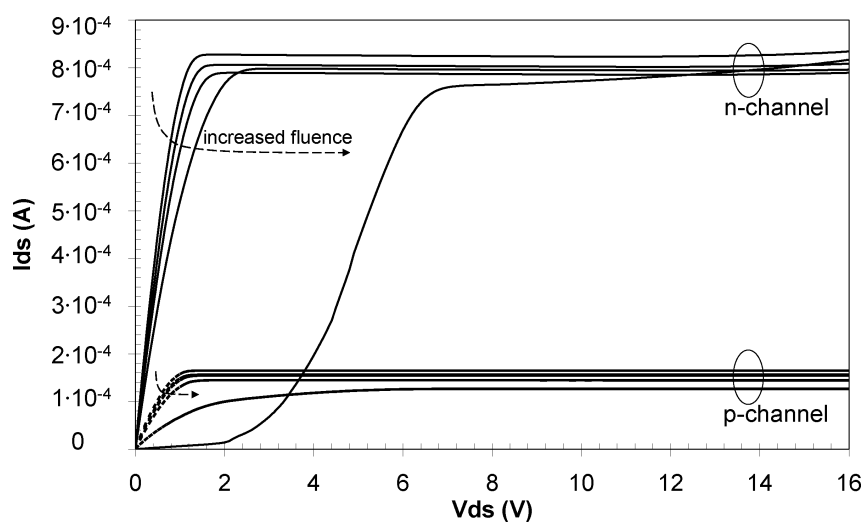

Fig. 14. Measured output characteristics for $\mathrm{Vgs}=1.8 \mathrm{~V}$ of long-channel $\mathrm{n}$ - and p-LDMOS transistors $(\mathrm{L}=10 \mu \mathrm{m})$ in technology $\mathrm{D}(0.18 \mu \mathrm{m})$ for increasing proton fluence (along the arrows: pre-rad, $5 \cdot 10^{14}, 1 \cdot 10^{15}, 2 \cdot 10^{15}$, $\left.6 \cdot 10^{15} \mathrm{p} / \mathrm{cm}^{2}\right)$.

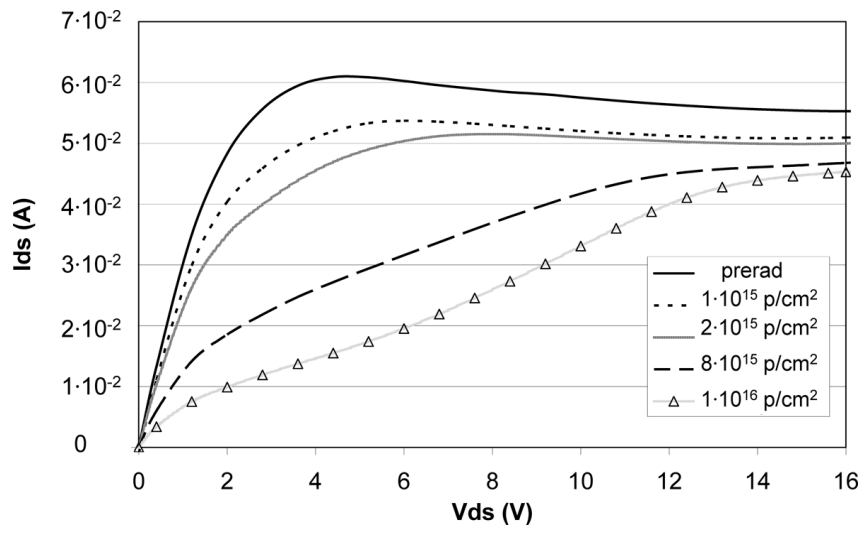

Fig. 15. Degradation of the output characteristics for $\mathrm{Vgs}=2.5 \mathrm{~V}$ induced by displacement damage on an n-channel LDMOS with HDB layout in the IHP $0.25 \mu \mathrm{m}$ technology (B).

this similar to its short channel counterpart in Fig. 12. Similar results were obtained for the samples in technology $\mathrm{E}$.

The ELT-type N-channel LDMOS designed in the $0.35 \mu \mathrm{m}$ technology (A) fails after proton irradiation, when a large drainbulk current can be measured at $\mathrm{V}_{\mathrm{ds}}$ as low as $2 \mathrm{~V}$ (the device is rated to $14 \mathrm{~V}$ in $\mathrm{V}_{\mathrm{ds}}$ ) already after an integrated fluence of $1 \cdot 10^{15} \mathrm{p} / \mathrm{cm}^{2}$. The HBD layout modification has evidently introduced a weakness in the high voltage capability of the transistor. When defects are introduced by displacement damage, the device is unable to stand the rated voltage anymore. Standard devices in the same technology (A), in fact, still perform correctly even after $5 \cdot 10^{15} \mathrm{p} / \mathrm{cm}^{2}$.

On the contrary, the alternative design implemented by IHP in their $0.25 \mu \mathrm{m}$ technology functions correctly even after proton irradiation, as evidenced by the very similar degradation in Fig. 15 (HBD layout) and Fig. 11 (standard design). In this case, the HBD modification has successfully reduced TID-induced leakage currents without penalizing the resilience to displacement damage effects. However, this design requires more area to achieve the same on-resistance (about 10-20\%) on a large area device.

\section{CONCLUSION}

An increasing number of semiconductor technology manufacturers are offering an additional high-voltage module to their 
standard mixed-mode CMOS processes. Vertical or, more frequently, lateral (LDMOS) transistors rated at drain-source voltages of $14-80 \mathrm{~V}$ are available for integrated circuits whose applications range from automotive to $\mathrm{RF}$, with large use in power management. The response of these high-voltage devices to cumulative radiation effects is systematically studied on samples from five different technologies in the 0.35 to $0.13 \mu$ m nodes.

Wide variability in TID response is observed. Threshold voltage shifts generally correlate with the gate voltage ratings of each device, hence with gate oxide thickness. On the contrary, no systematic correlation is observed for the source-drain leakage currents in $\mathrm{n}$-channel transistors. The leakage increase seems hence to be determined mainly by details in the construction of the device that are not necessarily linked to voltage ratings or technology node. It is noticeable that significant leakage occurs in all cases starting from a TID above 100 $\operatorname{krd}\left(\mathrm{SiO}_{2}\right)$ in the WC bias condition. Given the very limited $\mathrm{V}_{\text {th }}$ shift observed in all samples at this TID (with possibly only one exception), it is reasonable to expect that commercial off the shelf (COTS) components in these technologies could be suitable for a wide range of applications in Space-at least for cumulative effects.

When irradiated with high fluence of particles introducing displacement damage, both vertical and lateral transistors evidence serious electrical degradation. In particular, a large increase of the on-resistance is observed in all devices from a fluence that can vary considerably between technologies, but always above $5 \cdot 10^{14} \mathrm{p} / \mathrm{cm}^{2}$ (hence this is not an issue for typical Space applications). Measurements on LDMOS transistors also reveal a relevant deformation in the output characteristics, probably indicating that defects introduced by displacement damage affect the intimate structure of the device. The physical mechanism behind this degradation should be further investigated with the help of simulation tools, which was beyond the scope of this work.

HBD layout techniques inspired from those commonly used in low-voltage transistors are experienced for the first time in $\mathrm{n}$-channel high-voltage devices. In vertical and LDMOS transistors, they successfully extend the natural TID radiation tolerance, eliminating or (in one case) considerably reducing the TID-induced leakage current. These techniques can nevertheless be invasive in LDMOS transistors, and affect their high $\mathrm{V}_{\mathrm{ds}}$ voltage capability. In our study, an ELT LDMOS failed consistently to stand high $\mathrm{V}_{\mathrm{ds}}$ voltage after proton irradiation.

In view of the development of a custom DC-DC converter for the upgraded detectors at the CERN LHC collider, the IHP $0.15 \mu \mathrm{m}$ technology appears to better satisfy the requirements in terms of both TID (250 $\mathrm{Mrad}(\mathrm{SiO} 2)$ ) and displacement damage $\left(2.5 \cdot 10^{15} \mathrm{n} / \mathrm{cm}^{2}, 1 \mathrm{MeV}\right.$ neutron equivalent). The observed cumulative degradation of the LDMOS at these levels of radiation can be extrapolated to an efficiency drop below $5 \%$ for the converter, which is acceptable. The use of the other technologies would lead to a larger drop, and in some cases to the complete failure of the converter.

The assessment of the TID and displacement damage response of the LDMOS transistors should be complemented by the study of possible sensitivity of the devices to Single Event Effects (SEE) in a particle environment dominated by high energy hadrons. In particular, recent work on Single Event Burnout (SEB) indicated a significant influence of some LDMOS construction parameters on SEB threshold both in terms of $\mathrm{V}_{\mathrm{ds}}$ and ion Linear Energy Transfer (LET) [18]. Our work will hence be expanded to include the characterization of SEE sensitivity, for which a dedicated set of test structures is currently being fabricated. Measurements are planned using both heavy ions and high energy protons.

\section{ACKNOWLEDGMENT}

The authors would like to thank Dr. D. Knoll (IHP) and Dr. M. Ullan (IMB-CNM, CSIC, Barcelona, Spain) for their role in establishing the collaboration between CERN and IHP.

\section{REFERENCES}

[1] S. Orlandi et al., "Optimization of shielded PCB air-core toroids for high efficiency dc-dc converters," presented at the IEEE Energy Conversion Congress and Exposition, San Jose, CA, Sep. 2009.

[2] S. Buso, G. Spiazzi, F. Faccio, and S. Michelis, "Comparison of dc-dc converter topologies for future SLHC experiments," presented at the IEEE Energy Conversion Congress and Exposition, San Jose, CA, Sep. 2009.

[3] H. J. Barnaby, "Total-ionizing-dose effects in modern CMOS technologies," IEEE Trans. Nucl. Sci., vol. 53, pp. 3103-3121, Dec. 2006.

[4] R. Lacoe, "CMOS scaling, design principles and Hardening-by-Design methodologies," presented at the Short Course at the IEEE Nuclear and Space Radiation Effects Conference (NSREC), Monterey, CA, Jul. 2003.

[5] F. Faccio, "Radiation Issues in the new generation of high energy physics experiments," Int. J. High Speed Electronics and Systems, vol. 14, no. 2, pp. 379-399, 2004.

[6] M. Trivedi, P. Khandelwal, and K. Shenai, "Performance modeling of RF power MOSFET's," IEEE Trans. Electron Devices, vol. 46, no. 8, pp. 1794-1802, Aug. 1999.

[7] G. Anelli et al., "Radiation tolerant VLSI circuits in standard deep submicron CMOS technologies for the LHC experiments: practical design aspects," IEEE Trans. Nucl. Sci., vol. 46, pp. 1690-1696, Dec. 1999.

[8] L. J. Palkuti and J. J. LePage, "X-ray wafer probe for total dose testing," IEEE. Trans. Nucl. Sci., vol. NS-29, pp. 1832-1837, Dec. 1982.

[9] F. Gianotti et al., "Physics potential and experimental challenges of the LHC luminosity upgrade," Eur. Phys. J. C, vol. 39, pp. 293-333, Feb. 2005.

[10] P. W. Marshall and C. J. Marshall, "Proton effects and test issues for satellite designers," presented at the Short Course at the IEEE Nuclear and Space Radiation Effects Conf. (NSREC), Norfolk, VA, Jul. 1999, no. IVb.

[11] G. Lindström, M. Moll, and E. Fretwurst, "Radiation hardness of silicon detectors-A challenge from high-energy physics," Nucl. Instr. Meth. Phys. Res. A, vol. 426, pp. 1-15, Apr. 1999.

[12] F. Faccio and G. Cervelli, "Radiation-induced edge effects in deep submicron CMOS transistors," IEEE Trans. Nucl. Sci., vol. 52, pp. 2413-2420, Dec. 2005.

[13] F. Faccio et al., "Total ionizing dose effects in shallow trench isolation oxides," Microelectron. Rel., vol. 48, pp. 1000-1007, May 2008.

[14] D. L. Blackburn, T. C. Robbins, and F. Galloway, "VDMOS power transistor drain-source resistance radiation dependence," IEEE Trans. Nucl. Sci., vol. NS-28, pp. 4354-4359, Dec. 1981.

[15] N.S. Saks, M. G. Ancona, and J. A. Modolo, "Radiation effects in MOS capacitors with very thin oxides at $80{ }^{\circ} \mathrm{C}$," IEEE Trans. Nucl. Sci., vol. NS-31, pp. 1249-1255, Dec. 1984

[16] N. S. Saks, M. G. Ancona, and J. A. Modolo, "Generation of interface states by ionizing radiation in very thin MOS oxides," IEEE Trans. Nucl. Sci., vol. NS-33, pp. 1185-1190, Dec. 1986.

[17] S. C. Witczak, R. C. Lacoe, J. V. Osborn, J. M. Hutson, and S. C. Moss, "Dose-rate sensitivity of modern nMOSFETs," IEEE Trans. Nucl. Sci., vol. 52, pp. 2602-08, Dec. 2005

[18] P. E. Dodd et al., "Development of a radiation-hardened lateral power MOSFET for point-of-load applications," presented at the IEEE NSREC Conf., Dec. 2009, vol.56. 